\title{
HIPERTENSÃO EM PACIENTE AFRO-DESCENDENTE
}

\section{HYPERTENSION IN AN AFRO-DESCENDANT PATIENT}

\author{
Celso Amodeo ${ }^{1}$
}

\section{CASO CLÍNICO}

ALT, 64 anos, masculino, raça negra, casado com história de hipertensão arterial desde os 40 anos e sempre com pobre controle tensional. Há um ano vem apresentando cefaleia ocasional e fraqueza. Não apresenta nenhuma outra queixa cardiovascular e/ou neurológica. Nega história pregressa de cardiopatia ou doenças renais. Informa que nunca foi aderente ao tratamento devido aos efeitos adversos que observou com o uso de diuréticos sobre a função sexual. Atualmente está somente sob uso de aspirina $200 \mathrm{mg} /$ dia e sinvastatina $40 \mathrm{mg} / \mathrm{dia}$.

Ecocardiograma com uma fração de ejeção de $65 \%$ com moderada hipertrofia ventricular esquerda. ECG também confirma a presença de HVE com alterações inespecíficas da repolarização ventricular.

Seu perfil lipídico mostrava um LDL-C de $168 \mathrm{mg} / \mathrm{dL}$, um colesterol total de $239 \mathrm{mg} / \mathrm{dL}$, um HDL-C de $46 \mathrm{mg} / \mathrm{dL}$, e um TG de $239 \mathrm{mg} / \mathrm{dL}$. Sua ureia é de $36 \mathrm{mg} / \mathrm{dL}$ e a creatinina de $0,9 \mathrm{mg} / \mathrm{dL}$. A filtração glomerular calculada pelo $\mathrm{ClCr}$ foi de $109 \mathrm{~mL} / \mathrm{min} / 1,73 \mathrm{~m}^{2}$. A glicemia de jejum estava em $108 \mathrm{mg} / \mathrm{dL}$.

Não é tabagista e consome bebida alcoólica esporadicamente. Joga futebol ocasionalmente e não tem queixa de cansaço anormal durante a atividade física. Não pratica nenhum tipo de atividade física regular.

Tem história familiar positiva para infarto agudo do miocárdio (IAM) e acidente vascular cerebral (AVC) em familiares diretos abaixo de 55 anos.

Ao exame físico o paciente apresentava um índice de massa corporal (IMC) de $34.9 \mathrm{~kg} / \mathrm{m} 2$, PA de 150/100 e FC de 78 bpm.

Fundo de olho com discreto estreitamento arteriolar, mas sem exudatos ou hemorragias. Exame do aparelho cardiorrespiratório foi normal. Abdome globoso sem visceromegalias. Extremidades com pulsos presentes, simétricos e sem edemas.

\section{COMENTÁRIOS}

A hipertensão arterial na raça negra diferencia-se da hipertensão na raça branca nos seguintes aspectos: em geral, começa em idades mais precoces, com maior intensidade e com maior grau de envolvimento dos chamados órgãos alvo. Isso contribui para o dobro de acidentes vasculares e 50\% de aumento na mortalidade devido a doença isquêmica do coração. A Sociedade Internacional de Hipertensão em Negros em suas recomendações de tratamento da hipertensão em negros ressalta que tais recomendações devem ser específicas para o grupo negro e individualizada de acordo com o risco cardiovascular de cada paciente.

$\mathrm{Na}$ raça negra existe maior sensibilidade ao sal do que na raça branca devido a maior tendência a retenção renal de sódio. Diferenças étnicas no manuseio da sobrecarga de sódio pelos rins pode ser uma das explicações da hipertensão arterial primária na raça negra. Possíveis mecanismos seriam diferenças genéticas nos transportes transmembranas de íons, principalmente sódio, modificações dos canais de sódio na célula epitelial renal, atividade do sistema renina angiotensina e maior expressão de substâncias vasoativas, como por exemplo, a endotelina. Portanto, a resposta da sobrecarga de sal, tanto em normotensos quanto em hipertensos da raça negra é de maior sensibilidade, com maior resposta pressórica quando comparada com os indivíduos da raça branca.

Em relação à prescrição de medicamentos anti-hipertensivos é sabido que mais de 2/3 dos pacientes da raça negra hipertensos irão necessitar de pelo menos duas classes de medicamentos para atingirem as metas de controle de pressão arterial. Tanto os betabloqueadores quanto os inibidores da enzima de conversão da angiotensina (ECA) são menos ativos nos hipertensos negros. Entretanto, em associação com doses baixas de tiazídicos eles apresentam a mesma resposta que a associação dos diuréticos com antagonistas de cálcio ou bloqueadores do receptor AT1 da angiotensina 2. Portanto, não cabe a discussão de qual droga em monoterapia deverá ser escolhida para este paciente, mas sim quais medicamentos em associação deverão ser prescritos, levando-se em conta, além da potência anti-hipertensiva, os possíveis efeitos de classe sobre o metabolismo lipídico e glicídico desse paciente.

É sabido que hipertensos da raça negra, quando comparados aos hipertensos da raça branca, tem risco aumentado de lesão de órgão alvo (como insuficiência cardíaca, AVC e nefropatia) e comorbidades (como o diabetes). Pacientes afrodescendentes com hipertensão arterial também demonstram baixa resposta ao uso de bloqueadores do sistema renina angiotensina (IECA ou BRA) e apresentam melhor resposta ao uso de derivados tiazídicos associados aos antagonistas dos canais de cálcio, ou mesmo aos BRAs. A preferência do BRA em detrimento do IECA está no fato de maior risco de angioedema com IECA na raça negra. O bloqueio do sistema renina angiotensina neste paciente tem a propriedade de diminuir a resistência periférica à ação da insulina e preservação tecidual, principalmente função renal.

Outro aspecto a ser comentado é a necessidade de ação

1. Setor de Cardiopatia Hipertensiva, Dislipidemia e Aterosclerose, Universidade Federal de São Paulo (UNIFESP), São Paulo, SP, Brasil. 
mais agressiva no tratamento da dislipidemia deste paciente. Somente a sinvastatina não surtiu efeito no controle lipídico. Dentro de um quadro de dislipidemia com baixo HDL e alto LDL e TG, optou-se por iniciar o tratamento com uma estatina mais potente e com reconhecida capacidade de aumentar o HDL-colesterol, tendo também efeito na redução dos triglicérides.

Diante do risco cardiovascular e do nível de pressão arterial abaixo de 180/120 mmHg optou-se por manter-se a prescrição de ácido acetilsalicílico na dose de $200 \mathrm{mg} /$ dia.

\section{SEGUIMENTO DO PACIENTE}

As orientações referentes à mudança do estilo de vida, principalmente, no que se refere à reeducação alimentar para uma dieta pobre em gorduras saturadas e sal foram seguidas no início do tratamento (por um mês). A pressão arterial no primeiro mês estava em 135/88 mmHg e o paciente mostrou redução de peso em $3 \mathrm{~kg}$. Entretanto, nos retornos seguintes, o paciente já não estava aderente a dieta e não mostrava mais redução de peso, e a pressão arterial se mantinha elevada. Justificava que devido ao seu trabalho acabava comendo muito fora de casa em locais de "fast food".

Três meses após a primeira consulta o paciente mantinha o mesmo peso e a pressão arterial ainda estava acima das metas preconizadas para esse paciente. Entretanto, os lípides mostravam uma boa redução, mesmo com a não aderência às medidas higienodietéticas recomendadas. O colesterol total diminuiu para $186 \mathrm{mg} / \mathrm{dL}$ e o HDL-colesterol aumentou para $51 \mathrm{mg} / \mathrm{dL}$. Tal fato demonstra melhor resultado com estatinas mais potentes, mesmo naqueles pacientes não completamente aderentes à dieta e atividade física.

As doses do BRA foram dobradas e foi reforçado a necessidade de maior controle do estilo de vida, principalmente em relação à dieta e atividade física.

\section{REFERÊNCIAS RECOMENDADAS}

1. Park IU, Taylor AL. Race and ethnicity in trials of antihypertensive therapy to prevent cardiovascular outcomes: a systematic review. Ann Fam Med. 2007;5:444-52.

2. Douglas, JG, Bakris GL, Epstein M, et al. The Hypertension in African Americans Working Group. Management of high blood pressure in African Americans. Arch Intern Med. 2003;163:525-41.

3. Weir MR, Haynes DS. Hypertension in African Americans: a paradigm of metabolic disarray. Semin Nephrol. 1996;16:102-09.

4. Wilson PW, D'Agostino RB, Levy D, Belanger AM, Silbershatz H, Kannel WB. Prediction of coronary heart disease using risk factor categories. Circulation. 1998;97:1837-47.

5. Weir MR. Salt intake and hypertensive renal injury in African Americans: a therapeutic perspective. Am J Hypertens. 1995;8:635-44.

6. Morris RC, Sebastian A. Forman A, et al. Normotensive salt sensitivity: effects of race and dietary potassium. Hypertension. 1999;33:18-23.

7. Chobanian AV, Bakris GL, Black HR, et al; Joint National Committee on Prevention, Detection, Evaluation, and Treatment of High Blood Pressure. National Heart, Lung, and Blood Institute; National High Blood Pressure Education Program Coordinating Committee. Seventh report of the Joint National Committee on Prevention, Detection, Evaluation, and Treatment of High Blood Pressure. Hypertension. 2003;42:1206-52.

8. Sacks FM, Svetkey LP, Vollmer WM, et al; for the DASH-Sodium Collaborative Research Group. Effects on blood pressure of reduced dietary sodium and the Dietary Approaches to Stop Hypertension (DASH) diet. DASH-Sodium Collaborative Research Group. N Engl J Med. 2001;344:3-10.

9. Falkner B, Kushner H. Effect of chronic sodium loading on cardiovascular response in young blacks and whites. Hypertension. 1990;15:36-43.

10. Parmer RJ, Stone RA, Cervenka JH. Renal haemodynamics in essential hypertension. Racial differences in response to changes in dietary sodium. Hypertension. 1994;24:752-57.

11. Townsend MS, Fulgoni VL 3rd, Stern JS, Adu-Afarwuah S, McCarron DA. Low mineral intake is associated with high systolic blood pressure in the Third and Fourth National Health and Nutrition Examination Surveys: could we all be right? Am J Hypertens. 2005;18(2):261-69.

12. Hansson L, Zanchetti A, Carruthers SG, et al. Effects of intensive bloodpressure lowering and low-dose aspirin in patients with hypertension: Principal results of the Hypertension Optimal Treatment (HOT) randomized trial. HOT Study Group. Lancet. 1998;351:1755-62.

13. Ogden LG, He J, Lydick E, Whelton PK. Long-term absolute benefit of lowering blood pressure in hypertensive patients according to the JNC VI risk stratification. Hypertension. 2000;35:539-43.

14. Neal B, MacMahon S, Chapman N. Effects of ACE inhibitors, calcium antagonists, and other blood-pressure-lowering drugs: Results of prospectively designed overviews of randomized trials. Blood Pressure Lowering Treatment Trialists' Collaboration. Lancet. 2000;356:1955-64.

15. Saunders E. Tailoring treatment to minority patients. Am J Med. 1990;88(3):21S-23S.

16. Saunders E, Smith WB, DeSalvo KB, Sullivan WA. The efficacy and tolerability of nebivolol in hypertensive African American patients. J Clin Hypertens (Greenwich). 2007; 9:866-75.

17. ALLHAT Officers and Coordinators for the ALLHAT Collaborative Research Group. Major outcomes in high-risk hypertensive patients randomized to angiotensin-converting enzyme inhibitor or calcium channel blocker vs diuretic: The Antihypertensive and Lipid-Lowering Treatment to Prevent Heart Attack Trial (ALLHAT). JAMA 2002;288:2981-97.

18. Wright JT Jr, Dunn JK, Cutler JA, et al; ALLHAT Collaborative Research Group. Outcomes in hypertensive black and nonblack patients treated with chlorthalidone, amlodipine, and lisinopril. JAMA. 2005;293:1595-608.

19. Wright JT Jr, Harris-Haywood S, Pressel S, et al. Clinical outcomes by race in hypertensive patients with and without the metabolic syndrome: Antihypertensive and Lipid-Lowering Treatment to Prevent Heart Attack Trial (ALLHAT). Arch Intern Med. 2008; 168:207-17. 